\title{
LACK OF OVERSIGHT ON PROGRESSIVE LAWS FUELLING HIV
}

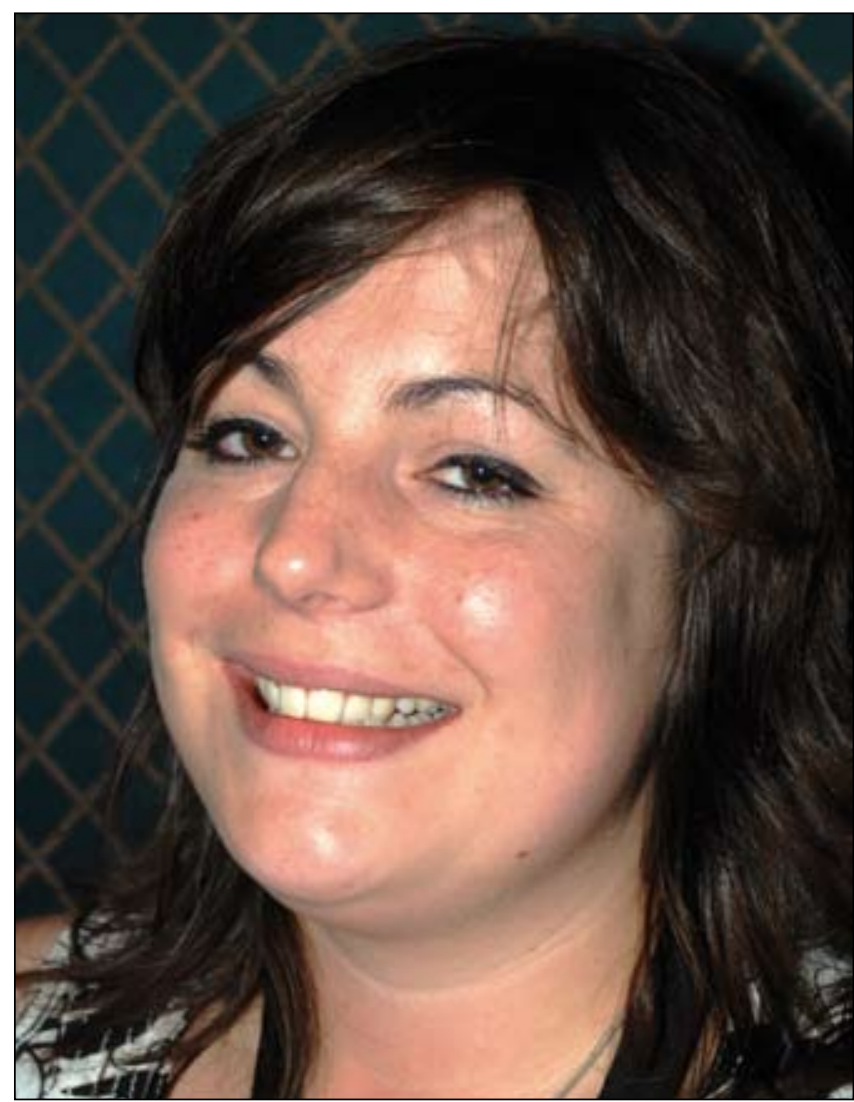

University of the Witwatersrand's Forced Migration Studies Programme researcher, Jo Vearey.

Picture: Chris Bateman

Ignorance of a 2007 Department of Health (DoH) directive to provide all foreign migrants with access to antiretroviral treatment, and understaffed and overburdened local facilities, have entrenched widespread ongoing discrimination and worsened health care outcomes.

This is according to two migration researchers who shared data on migrants' access to health and HIV prevention services at the 5th Social Aspects of HIV/AIDS Research Alliance (SAHARA) conference at Midrand in early December last year.

Ms Joanne Vearey of Witwatersrand University's Forced Migration Studies Programme and Ms Erin Tansey, Migration Health Project Officer for the International Organisation for

Vearey's research showed that many health care facility staffers genuinely believed they would 'get into trouble' if they catered for migrants - when the very opposite was legally and constitutionally required.
Migration based in Pretoria, said local rural migrants not in possession of their green ID book were also being victimised.

Vearey warned that if the DoH was basing its planning on existing 2001 census data 'then we have a big problem', adding that from clinicians to receptionists at many facilities in frontline provinces, migrant exclusion was the norm.

The main reason was that the $2007 \mathrm{DoH}$ directive was distributed in an ad hoc manner, often to district health offices where staff were asked to 'make copies and pass on' - meaning that where this happened the directive was often smudged and illegible.

There was no subsequent monitoring and evaluation by the DoH on the directives' impact or any training or followup to ensure that progressive and pragmatic South African legislation was actually carried out.

Vearey's research showed that many health care facility staffers genuinely believed they would 'get into trouble' if they catered for migrants - when the very opposite was legally and constitutionally required.

Tansey emphasised: 'If you're treating the migrant in the community, you're treating everyone'.

Said Vearey bluntly: 'The responsibility of the public sector is being met by NGO service providers'.

\section{'Casuals' get no employee health benefits}

She said that in the private sector a strong contributory factor to migrant exclusion was that where 'in-house' wellness programmes existed, they were for staff, not casual labour (i.e. mostly migrants).

Migrants also tended to avoid government clinics because of the long queues that they could not afford to stand in while battling to earn a living wage - and many feared deportation.

She recommended that government and corporate clinics be kept open after hours.

Vearey said local rural migrants were also often turned away at inner-city health facilities as a pragmatic means of shortening patient queues, adding 'it's not as simple as being xenophobic - the facilities are overburdened and understaffed'.

Both researchers said their data showed that nongovernmental organisations (NGOs) were trying to 'pick up the slack', but making a small impact on the overall problem. This 'dual health care' system where non-citizens were referred out of the public sector into the NGO sector (for either 'being 
foreign' or having no South African identity book) presented a range of challenges. These included cross-referrals, loss to follow-up, workload pressure and adherence.

Said Vearey bluntly: 'The responsibility of the public sector is being met by NGO service providers'.

Tansey said her research revealed that hundreds of 'problem' smaller companies who had been sub-contracted for the 2010 World Cup were failing to provide wellness programmes because they used mainly casual labour. She said 'very, very few programmes' in South Africa focused specifically on migrant labour.

One of the biggest contributors to the spread of HIV / AIDS was a lack of recreational facilities that would help assimilate migrants into communities and address the boredom and loneliness that led to drinking and sex, especially for truckers at border posts.

\section{Common assumptions on migrants debunked}

Vearey's research debunked several myths and assumptions about migrants.

Migration was economic and not linked to seeking health care, providing health care did not result in a 'flood of migrants', and migrant adherence to ART was actually better than that of local citizens.

Asked what contributed to higher adherence, Vearey told Izindaba that higher levels of social capital, better education and strong motivation for moving all played a role.

'There's a different profile on the person, they have a different energy around the importance of working and often a stronger support network,' she added.

Her research showed that non-citizens actually had fewer admissions to in-patient facilities, fewer of them missed appointments for ART initiation, they had a faster 'mean time' to ART initiation, better retention in care and lower overall mortality. Non-citizens were also less likely to fail ART than citizens.

\section{Problems experienced when trying to access public health services}

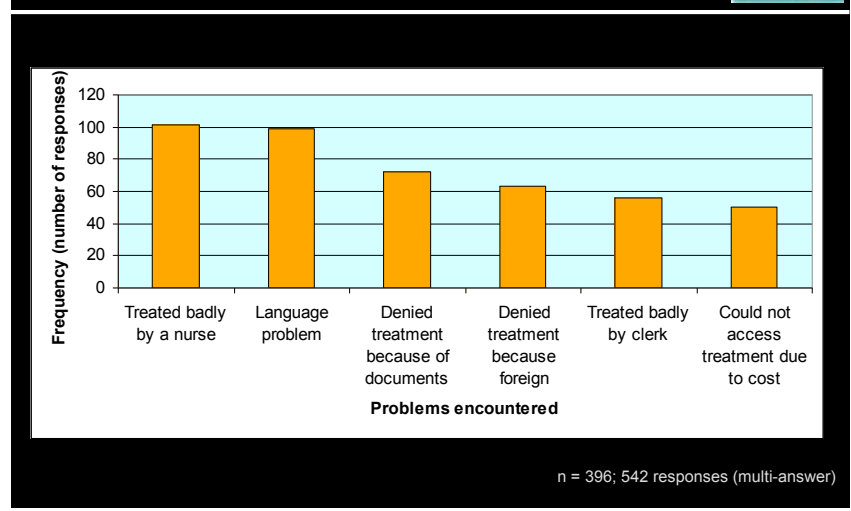

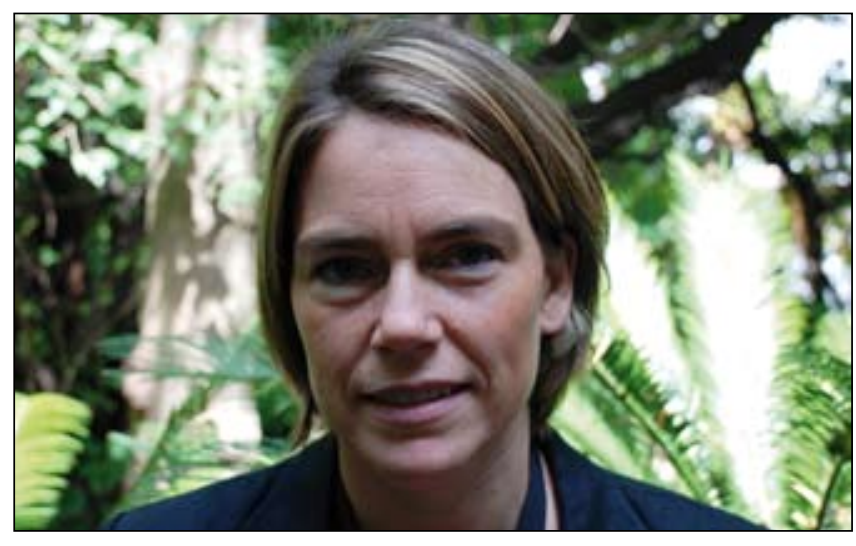

International Organisation for Migration, Health Project Officer, Erin Tansey.

Picture: Chris Bateman

Migrants reported that they would return home if they were too sick to work and that if a relative back home was sick they would not bring them to Johannesburg (where one particular study was conducted). Instead they would either send money home or return home themselves to care for them.

Vearey concluded that while the number of international migrants in need of health care and ART was small, it was 'significant'.

Existing protective legislation was not being applied uniformly across public institutions and the objectives of the National Strategic Plan (for HIV and Sexually Transmitted Infections) were not being implemented.

Upholding the right to health care for everybody in South Africa would have a population-level benefit.

\section{Pragmatic recommendations to government}

She recommended that government 'engage with and understand' migration and population growth and urged the implementation of a public health approach to migrants. More data were also needed to inform inter-sectoral, evidence-based regional policies.

Tansey's recommendations closely mirrored these, adding that projects and programmes need urgent developing to target 'places of vulnerability', as opposed to 'persons of vulnerability'.

Tansey said the Southern African Development Community (SADC) countries should standardise customs clearance procedures at border posts to reduce waiting times, co-ordinate accessible health facilities and HIV programmes regionally, and harmonise ART protocols.

She suggested introducing a card system that could be used within the region to access ART and recommended greater regulation of smaller companies or providing them with incentives to implement workplace policies that enabled greater access for all staff to HIV prevention services.

\section{Chris Bateman}

